\title{
Simultaneous Acute Achilles Tendon and Bilateral Quadriceps Tendon Rupture: a Rare Case Report
}

\author{
Ebrahim Zonozi ${ }^{1}$; Ali Jahansouz ${ }^{1, *}$ \\ ${ }^{1}$ Department of Orthopedic Surgery, Firouzgar Hospital, Iran University of Medical Sciences, Tehran, IR Iran \\ ${ }^{*}$ Corresponding author: Ali Jahansouz, Department of Orthopedic Surgery, Firouzgar Hospital, Iran University of Medical Sciences, Tehran, IR Iran. Tel: + 98-2133542010, Fax: + 98- \\ 2133542020, E-mail: a_jahansouz@yahoo.com \\ Received: November 28, 2014; Revised: January 1, 2015; Accepted: January 12, 2015
}

\begin{abstract}
Introduction: Quadriceps and Achilles tendon ruptures are well-known entity following tendonitis and tendon weakening in some of the chronic and systemic underlying disease.

Case Presentation: We report a very rare case with simultaneous Achilles and bilateral quadriceps tendon ruptures in a patient with chronic renal failure (CRF) in a 48-year-old man. The ruptured tendons were treated surgically with good outcome.

Conclusions: Multiple tendons injury should be considered to prevent delayed diagnosis in patients with CRF who presented with tendon rupture.
\end{abstract}

Keywords: Achilles Tendon; Quadriceps Tendon; Rupture; Renal Failure

\section{Introduction}

Quadriceps and Achilles tendons ruptures are wellknown entity following tendonitis and tendon weakening in some chronic and systemic underlying diseases (1). Quadriceps tendon rupture usually occurs in patients over 40 years with underlying disease. Some of the predisposing factors are diabetes, hyperparathyroidism, gout, chronic renal failure (CRF), rheumatoid arthritis (RA) and corticosteroid use. Quadriceps rupture originates in the tendon of the rectus femoris, often extending into the vastus intermedius tendon or transversely into the medial and lateral retinacula (2). Achilles tendon rupture is also predisposed to rupture. Aging, high body mass index (BMI) and chronic systemic diseases are the main causes (3). We report a patient with simultaneous bilateral Quadriceps and unilateral Achilles tendons rupture in a patient with underlying CRF.

\section{Case Presentation}

The situation of a 48-year-old man, known case of end stage renal disease (ESRD), under treatment with hemodialysis, was consulted with the orthopedic department. His chief complaint was inability to walk, after falling from two steps in hemodialysis ward since the morning of consultation. He had bilateral knee and right ankle pain. Skin had ecchymosis on the mentioned joints. Neurovascular examination for both lower extremities was normal. He was unable to extend both knees and there was a palpable gap in right an- kle, about $3 \mathrm{~cm}$ proximal to Achilles tendon insertion. Thompson squeeze test in right ankle was positive. Radiographs were normal, but according to both knee and right ankle magnetic resonance imaging (MRI) findings, bilateral quadriceps tendon rupture and right ankle Achilles tendon rupture were diagnosed (Figure 1). The quadriceps ruptures were both $4-5 \mathrm{~cm}$ proximal to upper pole of patella. He underwent bilateral quadriceps tendons repair by Krackow locking sutures passed through the holes drilled in the patella by nonabsorbable No.5 suture. The Achilles tendon rupture was also repaired by Krackow technique sutures in the same manner and reinforced by plantaris tendon. After three months of rehabilitation he was able to walk without assistance. Written consent of our patient was obtained to publish this report.

\section{Discussion}

Quadriceps tendon injury is usually the result of an eccentric overload to the extensor mechanism with the knee partially flexed (1). Systemic diseases such as diabetes, uremia, prior steroid injection (4), gout (5), pseudogout (5) and alkaptonuria (6) have been associated with quadriceps tendon rupture. Achilles tendon rupture has been estimated to be the third most frequent tendon rupture (1). Sudden unexpected ankle dorsiflexion or violent dorsiflexion of the plantar flexed foot can result in the rupture of this tendon. Aging, excessive femoral anteversion, muscle weakness has been reported as predisposing 

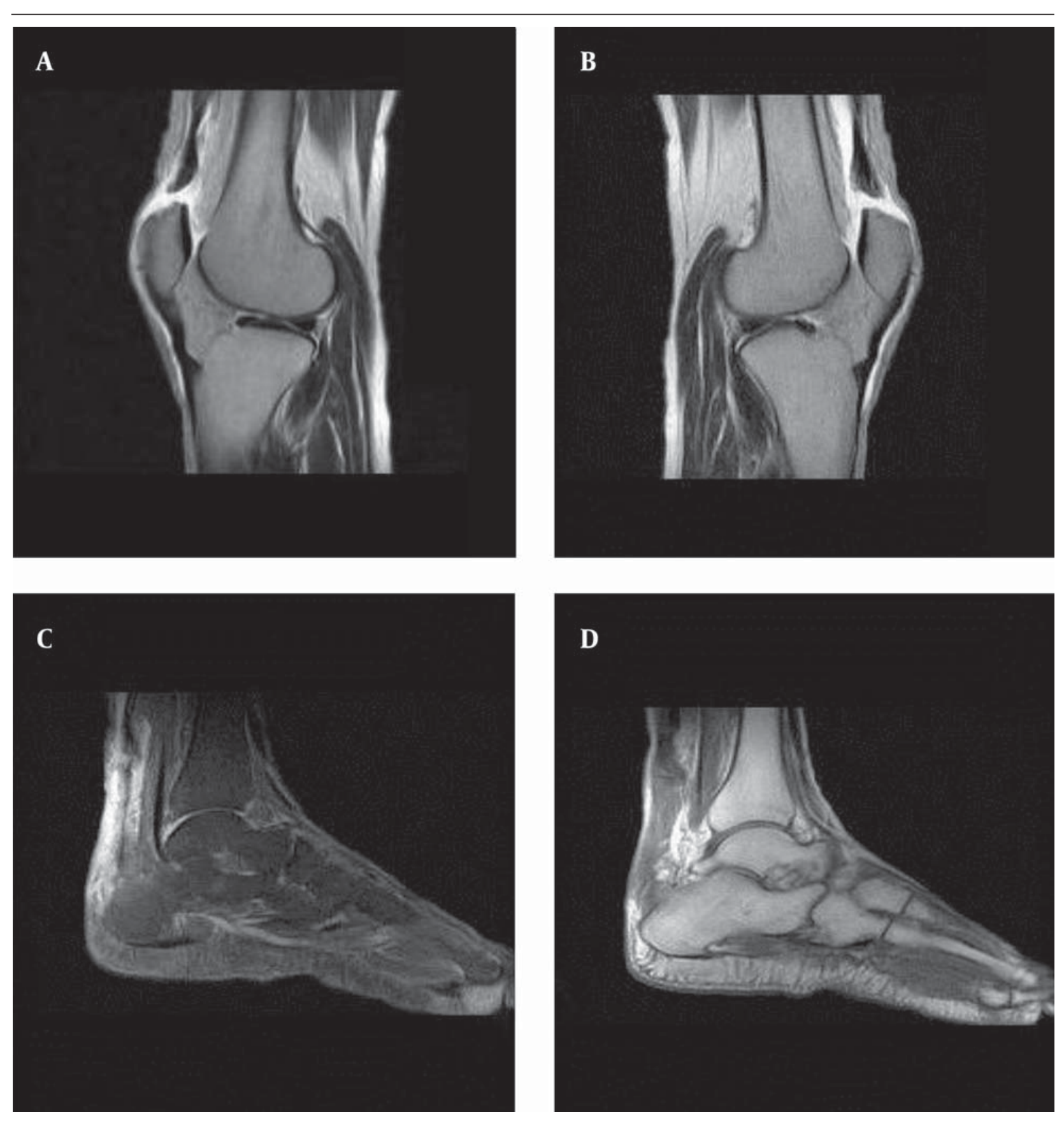

Figure 1. Quadriceps Tendons of (A) Right and (B) Left Knees and Achilles Tendon of the Ankle (C, D)

intrinsic risk factors for the injury (1). Bilateral simultaneous quadriceps tendon rupture is rare and described for the time by Steiner and Palmer in 1949 (7) and is thought to be the result of tendon weakening due to obesity and arteriosclerosis-induced fibrotic changes, or previous injury (7).

In chronic renal failure and chronic metabolic acidosis tendon collagen content alteration and elastosis result and predispose to tendon rupture (5). Plain X-ray, ultrasound and MRI are useful diagnostic tools which can help the clinician to establish the diagnosis (8). MRI is the most accurate method in diagnosing tendon ruptures and is particularly useful for preoperative planning because it allows better visualization of soft-tissues and anatomical details (9). Early surgical intervention is associated with optimal function. Delayed surgical repair has been associated with more difficult repair, longer rehabilitation, more post operation morbidity and use of adaptive equipment (10). Literature review identified only two published cases of this kind of injury. The first one has been published in 1978 reporting a patient with simultaneous bilateral quadriceps and 
Achilles tendons in one limb and of the patellar tendon of the other limb in a patient on chronic hemodialysis (11). This patient has undergone Para thyroidectomy six days before tends rupture (11). The other one was a case of bilateral and simultaneous quadriceps and Achilles tendons rupture in a 31-year-old male with the only risk factor of oral anabolic steroids consumption (12). Our case is the third one of reported simultaneous bilateral quadriceps tendon and Achilles tendon rupture of one limb.

In conclusion, because of rarity of bilateral Achilles or quadriceps tendon rupture the diagnosis is usually difficult and high grade of suspicion is essential for diagnosis of the injury properly. Complete and careful physical examination and paraclinic would be very helpful in making correct diagnosis and proper management.

\section{References}

1. Canale ST, Beaty JH. Campbell's Operative Orthopaedics: Expert Consult Premium Edition-Enhanced Online Features. 12th ed: Elsevier Health Sciences; 2012.

2. Scott WN. Surgery of the knee: Churchill Livingstone. 5th ed: Elsevier; 2012.
3. Hak DJ, Sanchez A, Trobisch P. Quadriceps tendon injuries. Orthopedics. 2010;33(1):40-6.

4. Liow RY, Tavares S. Bilateral rupture of the quadriceps tendon associated with anabolic steroids. Br J Sports Med.1995;29(2):77-9.

5. Ribbans WJ, Angus PD. Simultaneous bilateral rupture of the quadriceps tendon. BrJ Clin Pract. 1989;43(3):122-5.

6. Chua SY, Chang HC. Bilateral spontaneous rupture of the quadriceps tendon as an initial presentation of alkaptonuria--a case report. Knee. 2006;13(5):408-10.

7. Steiner CA, Palmer LH. Simultaneous bilateral rupture of the quadriceps tendon. Am J Surg. 1949;78(5):752-5.

8. Geisl H. [Bilateral simultaneous spontaneous and subcutaneous rupture of the quadriceps tendons]. Aktuelle Traumatol. 1983;13(5):201-4.

9. Barasch E, Lombardi LJ, Arena L, Epstein E. MRI visualization of bilateral quadriceps tendon rupture in a patient with secondary hyperparathyroidism: implications for diagnosis and therapy. Comput Med Imaging Graph. 1989;13(5):407-10.

10. Walker LG, Glick H. Bilateral spontaneous quadriceps tendon ruptures. A case report and review of the literature. Orthop Rev. 1989;18(8):867-71.

11. Fery A, Sommelet J, Schmitt D, Lipp B. Simultaneous bilatera avulsion of the quadriceps and Achilles tendons in 1 limb and of the patellar tendon in the other in a hyperparathyroid patient on chronic hemodialysis. Rev Chir Orthop. 1978;64(2):175-81.

12. Leong WY, Gheorghiu D, Rao J. A rare case of simultaneous acute bilateral quadriceps tendon rupture and unilateral achilles tendon rupture. J orthop. 2013;3(3):22-5. 